Printed in Great Britain

\title{
Spore Formation and 'Dimorphism' in the Mycobacteria
}

\author{
By ANNA CSILLAG \\ The Medical Research Council's Unit for Research on Drug Sensitivity in Tuber- \\ culosis, Postgraduate Medical School of London, Ducane Road, London, W. 12
}

(Received 9 February 1961)

\section{SUMMARY}

In studying 8 strains of Mycobacterium tuberculosis and 7 strains of atypical mycobacteria all 15 were found to produce, in addition to the typical acid-fast cells, non acid-fast ones, which gradually developed intracellular spore-like bodies; later free-lying spores were seen in the same cultures. This process occurred in heavily inoculated Löwenstein-Jensen medium cultures, which were at least 8 weeks old and were frequently aerated during incubation. With the atypical mycobacteria it occurred more readily in cultures in Kirschner fluid medium than on solid media. When the cultures containing spores were inoculated on nutrient agar plates, endospore-forming, rapidly growing organisms were obtained, which were not acidfast. These organisms when obtained from independent cultures of the same strain appeared to be identical in bacillary and colonial morphology at their first isolation on nutrient agar, but the organisms from different strains showed variation in these characters. Thus mycobacteria appear able to grow in two different forms: $(a)$ form 1 , which is acid-fast and multiplies by fission only; $(b)$ form 2, which is not acid-fast, produces endospores regularly and can be maintained in pure culture on nutrient agar. A series of phases of development of form 2 cells in the cultures of form 1 organisms in serial smear examination of Löwenstein-Jensen medium cultures is described. It is suggested that mycobacteria might be considered as dimorphic organisms in the same sense as some of the human pathogenic fungi are known to be dimorphic. Evidence is submitted that form 2 organisms are not contaminants.

\section{INTRODUCTION}

During experiments on the bacillary morphology of Mycobacteria (Csillag, 1960, 1961) and Nocardia asteroides, smears containing structures which resembled bacterial endospores or some sorts of fungal spores were occasionally seen. Such structures were found in the cultures of 17 of 24 strains of Mycobacterium tuberculosis, 20 of 42 strains of atypical mycobacteria (8 of group I, Runyon, 1959; 1 of group II; 7 of group III; 4 of group IV) and all of 4 strains of $N$. asteroides. When cultures containing these 'spores' were inoculated on nutrient agar plates, pure growths of spore-bearing bacilli or coccobacilli were obtained within 2 days, none of which were acid-fast. At first these organisms were considered as contaminants, but the possibility that they were derived from the acid-fast mycobacteria and nocardia was suggested by the following observations: (1) There were differences in the morphology of the spore-bearing, non acid-fast forms obtained from the different species of mycobacteria and nocardia. Thus, strains of $M$. tuberculosis always yielded Gram- 
negative organisms, whereas the atypical mycobacteria and nocardia strains yielded bacilli which were Gram-variable or Gram-positive. (2) The spore-bearing organisms obtained from different cultures of the same strains appeared to be identical. (3) The spore-bearing forms were isolated repeatedly in spite of precautions taken to avoid contamination. Systematic experiments were therefore carried out to establish whether these organisms were derived from, and represented a phase in, the life cycle of the acid-fast mycobacteria. The results are reported here. No systematic investigations have been done on the strains of $N$. asteroides. For purposes of convenience, the acid-fast mycobacteria are termed form 1 and the spore-bearing organisms, which are not acid-fast, form 2.

\section{METHODS}

\section{Mycobacterium tuberculosis}

Organisms used. Seven strains of I 968, I 971, I 977, I 1053, I 1122, I 1133, I 1155 were recently isolated from sputa of seven newly diagnosed and untreated British patients with pulmonary tuberculosis. The isolates were sensitive to isoniazid, streptomycin and $p$-aminosalicylic acid, and were fully virulent in the guinea pig shortly before the in vitro experiments were started. The isolation of these strains and the sensitivity tests were made by methods described elsewhere (Tuberculosis Chemotherapy Centre, Madras, 1959). One laboratory strain, H37 Rv. Atypical mycobacteria: Group I (Runyon, 1959; photochromogens): 4 strains (260, 266, 353, 1438). Group III (Runyon, 1959; Battey-type): 3 strains (223, 248, 585). All these seven strains were obtained from Dr E. H. Runyon (Veterans Administration Hospital, Salt Lake City, Utah, U.S.A.). They were all obtained from sputa, gastric contents or resected lung material of patients with pulmonary disease. All strains of $\boldsymbol{M}$. tuberculosis and atypical mycobacteria were maintained on Löwenstein-Jensen medium at $37^{\circ}$, and were subcultivated at intervals of a few months.

Media. (1) The Löwenstein-Jensen medium used was without potato starch (Jensen, 1955). (2) 7H-10 oleic acid-albumin agar plates (Cohn, Middlebrook \& Russell, 1959). (3) Kirschner medium (Mackie \& McCartney's Handbook, 1960) and Kirschner-base glycerol medium (containing $10 \%(\mathrm{v} / \mathrm{v})$ in place of $2 \%(\mathrm{v} / \mathrm{v})$ glycerol) dispensed in $3 \mathrm{ml}$. amounts in $\frac{1}{2}$-oz. screw-capped bottles. (4) Nutrient agar prepared by addition of $1 \cdot 4 \%(\mathrm{w} / \mathrm{v})$ agar to meat-extract + peptone broth (Oxoid No. 2, Oxo Ltd., London). All solid media were incubated at $37^{\circ}$ for 4 days and all fluid media for 5-6 days before use, as a test of their sterility.

Cultivation. All cultures were incubated at $37^{\circ}$. Plates of $7 \mathbf{H}-10$ medium and nutrient agar were sealed in polythene bags during incubation. All bacteriological manipulations were carried out in an inoculation cabinet.

Aeration of cultures. Provision of an additional air supply to the form 1 cultures growing on Löwenstein-Jensen medium was carried out as follows. The medium was normally dispensed in $3 \mathrm{ml}$. amounts in $\frac{1}{2}$-oz. screw-capped bottles, and incubated with the caps firmly on. Aeration was carried out by flaming the outside of the cap, loosening and flaming it again, removing the cap for a few seconds, flaming the neck of the bottle and replacing the cap. Slopes of Löwenstein-Jensen medium were also prepared in 'aeration tubes' which $\cos ^{2}$ isted of a test tube fitted with a rubber 
bung through which was passed a $3 \mathrm{~cm}$. diam. glass tube plugged with cotton wool. The tube was attached to $3 \mathrm{~mm}$. length of rubber tubing closed with a clip. The clip was opened for $30 \mathrm{~min}$. to allow the entry of air. During each batch of aeration a nutrient agar plate and a $5 \%$ horse blood agar plate were exposed near the hands of the worker in the inoculation cabinet in an attempt to cultivate airborne organisms.

Staining methods. Smears were allowed to dry under an ultraviolet lamp for 8-10 min., and fixed for $1 \mathrm{~min}$. in methanol. Jensen's method of Gram staining (Mackie \& McCartney, 1960) was modified by decolorizing rapidly with acetone and counterstaining with dilute (1/15) carbol fuchsin. Control smears of Staphylococcus pyogenes and Escherichia coli were usually stained on the same slide. In the ZiehlNeelsen staining (Mackie \& McCartney's Handbook, 1960) smears were decolorized with acid ethanol ( $\mathrm{NaCl} 20$ g.; distilled water, $500 \mathrm{ml}$; ethanol, 99.3\% (v/v), $1500 \mathrm{ml}$; $\mathrm{HCl}$ conc., $20 \mathrm{ml}$.) for not less than $5 \mathrm{~min}$. and counterstained with Loeffler's methylene blue for 5-10 min. The Loeffler's methylene blue was at least 5 weeks old. Staining for spores was done with warm $1 \%(w / v)$ malachite green in $1 \%(\mathrm{w} / \mathrm{v})$ phenol in water for $5 \mathrm{~min}$., rinsing in tap water for $3 \mathrm{~min}$., and counterstaining in $1 \%(\mathrm{w} / \mathrm{v})$ aqueous safranin for $30 \mathrm{sec}$.

\section{RESULTS}

\section{Production of form 2 mycobacteria}

Origin of form 2 from form 1 organisms. The following experiment was carried out to obtain form 2 organisms from cultures of form 1 mycobacteria. A total of 15 strains (8, Mycobacterium tuberculosis; 4, group I atypical; 3, group III atypical) were plated out on $7 \mathrm{H}-10$ medium plates and, after incubation for 17 days, single colonies were plated out again. With each strain, 6 slopes of Löwenstein-Jensen medium were each heavily inoculated from a separate well-isolated colony grown on the second plate for 17 days. An equal number (90) of Löwenstein-Jensen medium slopes from the same batch of medium were 'inoculated' in a similar manner, but with a sterile loop. These control slopes were subsequently subjected to exactly the same procedures as their counterpart slopes which had been inoculated with living organisms. The slopes were inoculated in a random order.

Groups of slopes containing one slope inoculated with a colony from each strain and an equal number of control slopes inoculated with a sterile loop were subjected to different procedures which are set out diagrammatically in Fig. 1. The slopes of groups $A 1$ and $B 1$ were in aeration tubes, while all the remaining slopes were in screw-capped bottles. The procedures for groups A and A 1 were otherwise identical, and similarly for groups B and B1. All cultures were incubated for 14-24 weeks. In groups $\mathrm{A}, \mathbf{A} 1, \mathrm{~B}, \mathrm{BI}$ and $\mathrm{C}$ the cultures were aerated for portions of the incubation period, with the aim of investigating the relationship between aeration and the occurrence of form 2 organisms. Cultures of group D were not aerated. At 14 weeks cultures of group B 1 were subcultured on to fresh Löwenstein-Jensen medium slopes (group S) and, after incubation for 8 weeks, the growth from group S was again subcultured into tubes of Kirschner and Kirschner-base glycerol medium (groups $\mathrm{K}$ and $\mathrm{Kg}$ ). Further control slopes or tubes inoculated with a sterile loop were also included in groups $\mathrm{S}, \mathrm{K}$ and $\mathrm{Kg}$. 
At the times indicated in Fig. 1, samples of the initial inoculum and of the growth in the cultures were inoculated in two nutrient agar plates and examined in smears stained by the Ziehl-Neelsen methods. The nutrient agar plates were incubated for 5 days and colonies were examined and smears were made from them daily. After the end of the experiment each Löwenstein-Jensen medium culture in group $\mathbf{C}$ was reincubated for a further 3 months and was aerated twice weekly. At the end of this period smears were stained from the growths on these slopes.

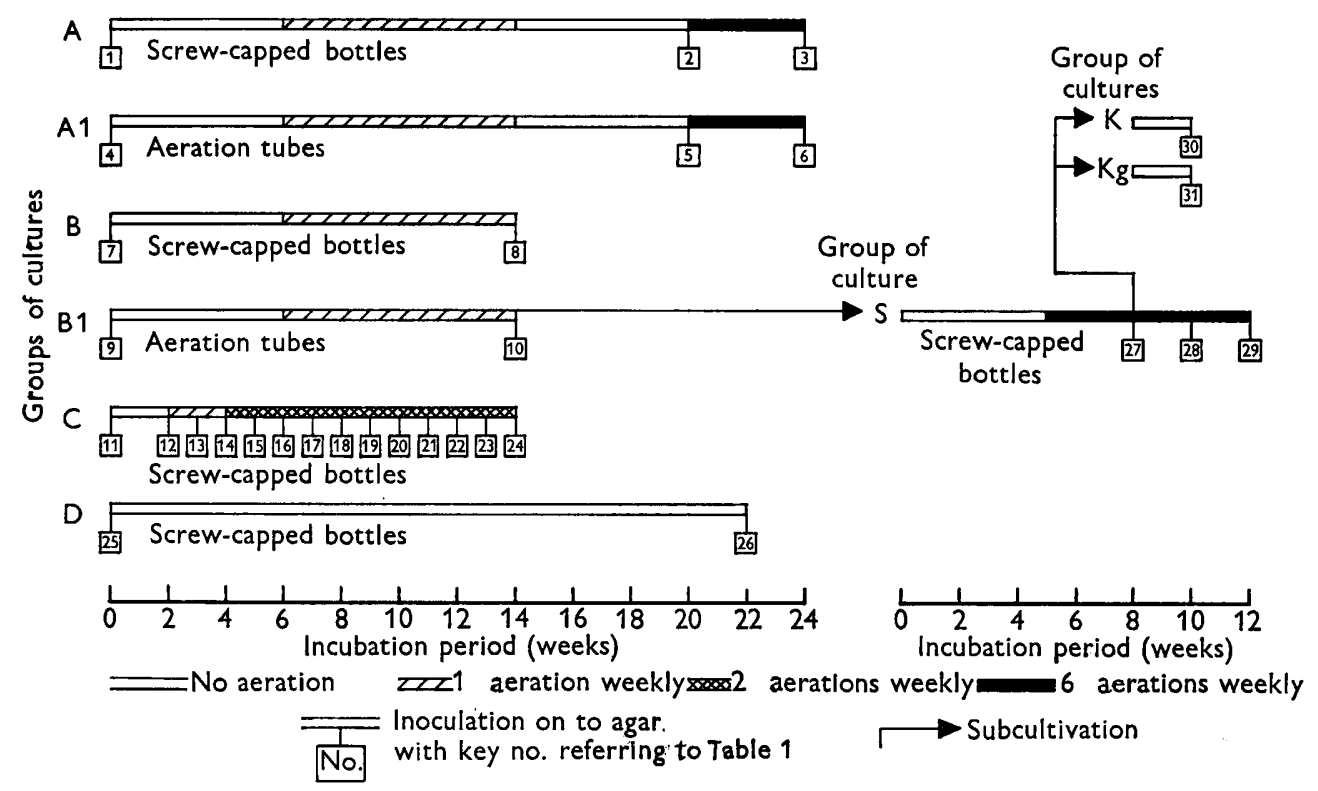

Fig. 1. Incubation and aeration of form 1 mycobacteria cultures.

The results of the cultures on nutrient agar plates inoculated at the intervals indicated in Fig. 1, from the growths on Löwenstein-Jensen and Kirschner media, are set out in Table 1. All samples of the colonies on $7 \mathrm{H}-10$ medium plates used for inoculating the Löwenstein-Jensen medium slopes failed to yield growth when inoculated on to nutrient agar plates. All 15 strains yielded form 2 colonies on nutrient agar plates on 3 to 10 occasions of sampling. Form 2 colonies were obtained only from form 1 cultures on Löwenstein-Jensen medium which had been incubated for 8 weeks or more. An isolation from a culture was not always followed by further isolations from the same culture, possibly because the samples were usually taken from a small area on the slope and were not representative of the entire growth. At each isolation a heavy growth of at least 100 form 2 colonies was obtained on incubation of the nutrient agar plates for 1-2 days. None of the control slopes, which had been initially inoculated with a sterile loop yielded any growth on the nutrient agar plates. None of the cultures of group D, which were not aerated, yielded form 2 colonies, when they were sampled at 22 weeks. However, form 2 colonies were obtained on each occasion of sampling at 8-14 weeks from 3 to 8 cultures in group $\mathrm{C}$, which were being aerated twice weekly.

Of the 88 pairs of nutrient and blood agar plates exposed for cultures of airborne 
Table 1. Isolation of form 2 organisms from form 1 mycobacteria

\begin{tabular}{|c|c|c|c|c|c|c|c|c|c|c|c|c|c|c|c|c|c|}
\hline \multirow{3}{*}{$\begin{array}{l}\text { Group } \\
\text { of } \\
\text { cultures }\end{array}$} & \multirow{3}{*}{$\begin{array}{l}\text { Period } \\
\text { of incu- } \\
\text { bation } \\
\text { (weeks) }\end{array}$} & \multirow{3}{*}{$\begin{array}{l}\text { Key } \\
\text { no. } \\
\text { from } \\
\text { Fig. } 2\end{array}$} & \multirow{2}{*}{\multicolumn{8}{|c|}{ M. tuberculosis }} & \multicolumn{7}{|c|}{ Atypical mycobacteria } \\
\hline & & & & & & & & & & & \multicolumn{4}{|c|}{ Group I } & \multicolumn{3}{|c|}{ Group III } \\
\hline & & & $\begin{array}{r}\text { H37 } \\
\text { Rv }\end{array}$ & $\begin{array}{c}\text { I } \\
968\end{array}$ & $\begin{array}{c}\text { I } \\
971\end{array}$ & $\underset{977}{I}$ & $\begin{array}{c}I \\
1053\end{array}$ & $\underset{1122}{I}$ & $\underset{1133}{I}$ & $\underset{1155}{I}$ & 260 & 266 & 353 & $\widetilde{1438}$ & 223 & 248 & 585 \\
\hline \multirow[t]{3}{*}{$\mathbf{A}$} & $\mathbf{0}$ & 1 & - & - & - & - & - & - & - & - & - & - & - & - & - & - & - \\
\hline & 20 & 2 & - & - & - & - & - & - & - & - & - & - & - & - & - & - & - \\
\hline & 24 & 3 & + & - & - & - & - & - & + & + & - & - & - & - & - & - & - \\
\hline \multirow[t]{3}{*}{ A 1} & 0 & 4 & - & - & - & - & - & - & - & _- & - & _ & - & - & _- & _- & _ \\
\hline & 20 & 5 & - & - & - & - & - & - & - & - & - & - & - & - & - & - & - \\
\hline & $\mathbf{2 4}$ & 6 & - & + & + & - & - & - & + & + & - & - & - & - & - & - & - \\
\hline \multirow[t]{2}{*}{ B } & $\mathbf{0}$ & 7 & - & - & - & - & - & - & - & - & - & - & - & - & - & - & - \\
\hline & 14 & 8 & - & + & + & + & - & + & + & - & - & - & - & - & - & - & + \\
\hline \multirow[t]{2}{*}{ B 1} & o & 9 & - & - & - & - & - & - & - & - & - & - & - & - & - & - & - \\
\hline & 14 & 10 & + & + & + & + & - & + & + & - & - & - & - & - & - & - & + \\
\hline \multirow[t]{14}{*}{ C } & $\mathbf{0}$ & 11 & - & - & - & - & - & - & - & - & - & - & - & - & - & - & - \\
\hline & $\mathbf{2}$ & 12 & - & - & - & - & - & - & - & - & - & - & - & - & - & - & - \\
\hline & $\mathbf{3}$ & 13 & - & - & - & - & - & - & - & - & - & - & - & - & - & - & - \\
\hline & 4 & 14 & - & - & - & - & - & - & - & - & - & - & - & - & - & - & - \\
\hline & 5 & 15 & - & - & - & - & - & - & - & - & - & - & - & - & - & - & - \\
\hline & 6 & 16 & - & - & - & - & - & - & - & - & - & - & - & - & - & - & - \\
\hline & 7 & 17 & - & - & - & - & - & - & - & - & - & - & - & - & - & - & - \\
\hline & 8 & 18 & + & - & - & - & - & - & - & - & - & - & - & + & - & + & - \\
\hline & $\mathbf{9}$ & 19 & + & - & - & - & - & + & + & - & + & - & + & + & + & + & - \\
\hline & 10 & 20 & - & - & - & - & - & + & - & - & + & - & + & + & - & - & - \\
\hline & 11 & 21 & - & + & - & - & + & - & - & + & - & - & - & + & + & + & - \\
\hline & 12 & 22 & - & - & - & + & - & + & - & - & + & - & - & + & - & - & - \\
\hline & 13 & 23 & + & - & - & - & - & + & - & - & - & + & + & + & - & - & - \\
\hline & 14 & 24 & - & - & + & - & + & - & - & - & + & - & + & + & - & - & + \\
\hline \multirow[t]{2}{*}{ D } & 0 & 25 & - & - & - & - & - & - & - & - & - & - & - & - & - & - & - \\
\hline & 22 & 26 & - & - & - & - & - & - & - & - & - & - & - & - & - & - & - \\
\hline \multirow[t]{3}{*}{$\mathbf{S}$} & 8 & 27 & - & - & - & - & - & - & - & - & - & - & - & - & - & - & - \\
\hline & 10 & 28 & - & - & - & - & + & - & - & - & - & - & - & + & + & - & - \\
\hline & 12 & 29 & + & + & - & - & - & - & - & - & - & - & + & + & + & - & - \\
\hline $\mathbf{K}$ & 2 & 30 & - & - & - & - & - & - & - & - & - & + & - & - & - & - & + \\
\hline KG & 2 & 31 & - & - & - & - & - & - & - & - & + & + & + & + & + & - & + \\
\hline
\end{tabular}

contaminants during the aeration of the cultures, only two plates yielded growth on subsequent incubation, and the colonies on both consisted of Gram-positive cocci.

After incubation for 2 days, plates bearing form 2 colonies of the first isolate from each strain were formolized for comparison with the other isolates. The bacillary morphologies of the form 2 organisms were compared in smears, stained by the Ziehl-Neelsen methods, when endospores first become apparent, that is, after the nutrient agar plates had been incubated for 1-5 days. Both the bacillary and colonial morphologies of the form 2 organisms, isolated from the same form 1 strain on different occasions from the same culture and from different cultures appeared identical. The similarity of the bacillary morphologies of five pairs of form 2 
organisms, each member of the pair being obtained from a different group, and the variation from strain to strain are illustrated in Pl. 1, figs. 2-6.

The effect of aeration. Aeration of the Löwenstein-Jensen medium cultures appeared to be necessary for the development of the form 2 organisms in the form 1 cultures, as seen with the cultures of group $\mathrm{D}$, which were not aerated and did not yield form 2 colonies. It also appears probable that the interruption of aeration resulted in the form 2 organisms losing their ability to grow on nutrient agar. In groups B and B 1 at 14 weeks, following an 8-week period of aeration form 2 colonies were obtained from $\mathbf{1 3}$ of the $\mathbf{3 0}$ cultures. However, cultures in groups A and A1 were aerated in the same way until the 14th week; they were not aerated for 6 weeks, when sampling failed to yield any form 2 colonies, but after a further period of intensive aeration 7 of the 30 cultures yielded these colonies. Subculturing followed by a period of growth without aeration also appeared to prevent the isolation of form 2 colonies. Colonies were obtained from 7 of the 15 cultures in group B1 at 14 weeks, when they were subcultivated as group S. After growth without aeration for 5 weeks and with aeration for a further 3 weeks, none of the cultures of group $S$ yielded form 2 colonies.

The effect of different media. The effect of different media on the development of form 2 mycobacteria appeared to differ according to whether the form 1 strains were Mycobacterium tuberculosis or atypical mycobacteria. Form 2 colonies were obtained from sampling of 7 of the 8 strains of $M$. tuberculosis, but from only 1 of the 7 strains of atypical mycobacteria in the Lowenstein-Jensen cultures of groups A, A 1, B and B1. Again, the subcultures in Kirschner-base glycerol medium (group $\mathrm{Kg}$ ) yielded form 2 colonies from none of the 8 strains of $M$. tuberculosis, but from 6 of the 7 strains of the atypical mycobacteria.

\section{Morphology of form 2 organisms in form 1 cultures}

Phases of development. The phases of development of form 2 organisms are described as they appeared in the cultures of group C (Table 1), since in this group smears were made at weekly intervals. Samples of the initial inoculum and of the growth in the cultures were stained by the Ziehl-Neelsen method. The microscopic appearances are illustrated diagrammatically in Fig. 2, since the early phases were difficult to see and even more difficult to photograph. Some of the phases appeared together in the smears and it is therefore difficult to be certain that the order of development is exactly as described. Considerable variation occurred from culture to culture in the time intervals between the occurrence of the phases, and only the average time is reported.

Form 2 cells first appeared between the fourth and sixth week of incubation as just visible non acid-fast dots lying free among the form 1 bacilli (Fig. 2, phase 1). At about 6 weeks short rods with one pointed end and a polar granule at the other, appeared, giving the impression of a germination tube (Fig. 2, phase 2). At about 8 weeks, slender rods with pointed ends were seen, and these were either stained evenly or contained a single deeper stained granule located centrally, or two granules located at the poles (Fig. 2, phase 3). A minority of these cells were arranged parallel to each other. Between 9 and 14 weeks the intracellular granules often became larger than the rest of the cell, distorting the cell wall and giving the impression of an early stage of intracellular spore-formation. At 14 weeks a pro- 
portion of the cultures yielded smears which contained either one or both of the following forms: $(a)$ evenly and deeply stained rods with squared ends, often lying in chains or parallel (Fig. 2, phase $4 a$ ); (b) free-lying oval spores, either isolated or in clumps (Fig. 2, phase $4 b$ ). An example of phase $4 b$ is shown in Pl. 1, fig. 1. All of the cultures in group $\mathrm{C}$ (Table 1 ) at the end of the additional 3-month period of incubation and aeration yielded smears containing organisms in phases $4 a$ and $b$.
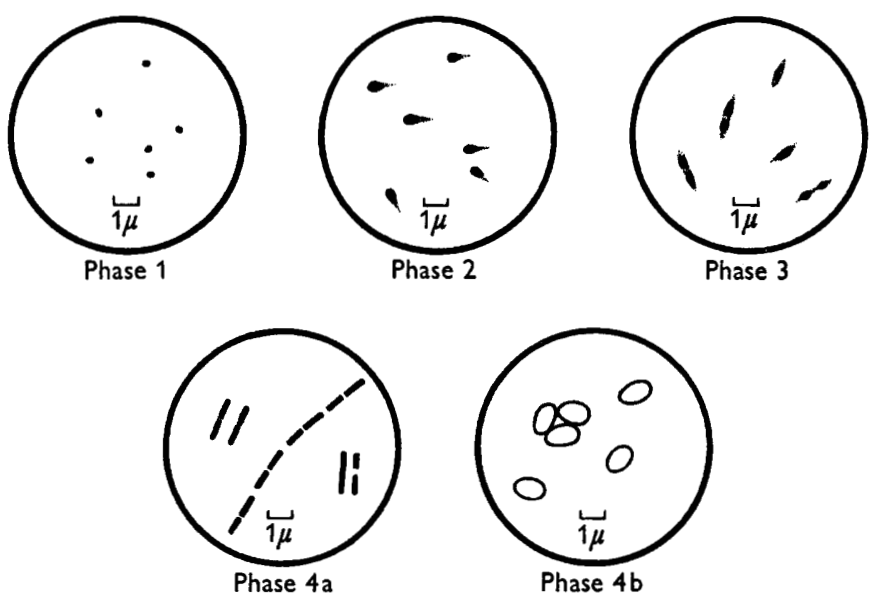

Fig. 2. Diagrammatic illustrations of development of form 2 organisms in form 1 cultures.

Form 2 organisms were stained in their early phases with difficulty, and did not stain with an aqueous solution of methylene blue or with Loeffler's methylene blue when the latter was less than 5 weeks old. The vegetative forms were never acid-fast. The central area of the free spores was usually unstained, but occasionally weakly acid-fast; the walls were stained blue. The spores stained green with safranin-malachite green. The number of the form 2 organisms in proportion to the number of form 1 organisms was highly variable. In phases 1 and 2 , form 2 organisms were present only sparsely and could easily be overlooked. When in phase 3 , the ratio of their number to the number of form 1 organisms was not less than $1: 100$.

Changes in the culture medium. The Löwenstein-Jensen medium did not alter until the form 2 organisms were in phases 1 and 2 ; when they were about to change from phase 3 to phases $4 a$ and $4 b$ the medium became yellowish, particularly at the edges of the slopes. When form 2 organisms reached phase 4, the growth often developed a brownish pigmentation or liquefied the medium, giving the impression that it was contaminated.

Ability to grow on nutrient agar. Form 2 organisms in phases 1, 2 and early in phase 3 did not grow on nutrient agar. In phase 3 , when the granules became large enough to distort the cell wall, and in phase $4 a$ and $4 b$, colonies were invariably obtained on nutrient agar plates. Form 2 organisms in phases 1,2 and early 3 disappeared from smears and lost their ability to grow on nutrient agar when aeration of the Löwenstein-Jensen medium cultures was interrupted for a long period, as described above. Once form 2 organisms reached phase 4, they were not 
influenced by interrupting the aeration, as shown by a further experiment in which cultures contained form 2 organisms in phase $4 b$ and were incubated without aeration for 12 months. The spores remained unchanged and yielded colonies on nutrient agar.

Growth on Löwenstein-Jensen medium. When form 1 cultures containing form 2 organisms in phases 1-3 were subcultured to fresh Löwenstein-Jensen medium, normal growth of form 1 organisms appeared and the growth did not contain form 2 organisms. However, when form 1 cultures on Löwenstein-Jensen medium, containing form 2 organisms in phases 4 , were subcultured on the same medium, the medium of the subculture turned yellow or brown and was often softened. Form 1 organisms never grew on it and at most, scanty colonies of form 2 were obtained on it.

\section{Properties of form 2 organisms isolated on nutrient agar}

Bacillary morphology. The bacillary morphology of the form 2 organisms is described from smears prepared from the colonies of the first isolate on nutrient agar. The appearance in smears stained by the Ziehl-Neelsen method are described when endospores first became apparent (1-5 days). Gram staining is described in smears taken at 2 days, irrespective of sporulation. Staining with safranin-malachite green was done when the cultures contained numerous free spores.

The bacillary morphology of individual strains is not described in detail, because further experience suggests that it is profoundly affected by the nature of the medium, the period of incubation, the age and previous history of the inoculum, and the temperature of incubation. Nevertheless, it is important to emphasize that the bacillary morphology of the different isolates from the same form 1 strain always appeared identical at the first isolation on nutrient agar. The form 2 organisms obtained from 12 strains were rods (Pl. 1, figs. 2, 3, 5, 6), which varied from strain to strain in their size (range $0.5 \times \mathbf{3} \cdot 0 \mu-1 \cdot 0 \times \mathbf{2} \cdot 5 \mu$ ), in the shape of their ends, in the arrangement of cells, in the location of their endospores and in the extent to which the spores distorted the cell walls. None of the rods was acid-fast. The rods from $M$. tuberculosis were Gram-negative, those from the atypical mycobacteria were Gram-variable or Gram-positive. After longer periods of incubation (3 days2 weeks) small, Gram-positive granules appeared within some of the rods from all strains. The free spores were oval (size range $0.7 \times 1.0 \mu-1.2 \times 1.5 \mu$ ), their cell walls were blue in the Ziehl-Neelsen smears and Gram-positive, the central area was usually unstained, but occasionally weakly acid-fast and Gram-negative. They were uniformly green when stained with safranin-malachite green. The form 2 organisms obtained from the remaining three strains (H37 Rv, I 977, I 1133) were cocci of very uneven size and shape, often arranged in chains (Pl. 1, fig. 4), which were not acidfast and were Gram-negative. The large, oval forms seen in Pl. 1, fig. 4, were not as typical of spores as those seen in the rod-shaped form 2 organisms, but they were still considered as spores since they stained green with safranin-malachite green. These spores stained uniformly Gram-negative. Although these coccoid forms were obtained from all of the $\mathbf{3}$ strains at all isolations, it has been observed that strain H37 Rv yielded rod-shaped form 2 organisms, with typical endospores after prolonged incubation and aeration on Löwenstein-Jensen medium. This finding suggests that the coccoid form may be an intermediate stage followed by a final rod-shaped stage similar to the remaining strains of $M$. tuberculosis. 
Viability. The form 2 organisms which were composed of cocci all died out after 1-2 subcultivations on nutrient agar. With the remaining strains, subcultures of colonies which did not contain free spores had a similar poor viability. When free spores were present, subcultures on nutrient agar could often be maintained in a viable state at room temperature for 2 months, but during regular subcultures at intervals of 2 months for a year most have died out.

Colonial morphology. The morphology of the colonies of the form 2 organisms is described from the first isolate from each strain obtained on nutrient agar plates after incubation for 2 days. The colonies were examined at $\times 10$ magnification with a plate microscope. As with bacillary morphology, no attempt has been made to describe the detailed colonial morphology of each strain since the appearances were markedly variable on further subcultures. Each strain, however, yielded the same colonial type when first isolated from the Löwenstein-Jensen medium slopes. The colonies obtained from the Mycobacterium tuberculosis strains (Pl. 2, figs. 1, 2) were usually small $(0 \cdot 1-0 \cdot 9 \mathrm{~mm}$. diam.), but larger colonies $(2 \cdot 0-3 \cdot 0 \mathrm{~mm}$. diam.) were obtained from three strains. The colonies were usually discrete, but one strain produced a confluent thin film on the surface of the medium; they were circular, or irregular, effuse or raised, smooth or finely granular; some strains had colonies with one central umbo, or two umbos or were umbilicate; the edges were usually entire though the large colonies had crenated or slightly fimbriate edges; all colonies were grey and opaque, most were friable, but the larger colonies were butyrous; one strain had colonies firmly adherent to the medium. The colonies obtained from the atypical mycobacteria were larger $(3 \cdot 0-8 \cdot 0 \mathrm{~mm}$. diam.); they were circular or irregular, smooth, glistening, butyrous either with a uniform structure and entire edges (P1. 2, figs. 3, 4) or a beaten-copper, worm-cast surface with rhizoid edges (Pl. 2, fig. 5).

\section{DISCUSSION}

Evidence that the form 2 organisms were derived from the form 1 organisms and were not contaminants can be derived from the following observations. (1) There was variation in the bacillary and colonial morphologies of form 2 organisms from strain to strain and from species to species, yet these characters were closely similar in multiple isolations from the same form 1 strain. It will be appreciated that the form 1 cultures in groups A, A 1, B, B 1 (in this context B 1 includes its subcultures $\mathrm{S}, \mathrm{K}$ and $\mathrm{Kg}$ ) and $\mathrm{C}$, were each derived from a separate colony on $7 \mathrm{H}-10$ medium plates (Fig. 1). The similarity of the bacillary morphologies of five pairs of form 2 organisms, each member of the pair being obtained from a different group and the variation from strain to strain are illustrated in Pl. 1, figs. 2-6. Some of the strains of Mycobacterium tuberculosis and atypical mycobacteria were again examined for the production of form 2 organisms in further experiments. The form 2 isolates had colonial and bacillary morphologies identical to those described here. (2) The conditions under which form 2 organisms were obtained, such as length of incubation and type of medium, were different with strains of $M$. tuberculosis and of the atypical mycobacteria. If the form 2 organisms arose as airborne or medium contaminants then a random pattern of these characters would be expected and would not be related to the species of the form 1 strains; however, the observations reported provide strong evidence against this view, particularly since the inoculation and aeration of the cultures was done in a random order. (3) The controls incorporated 
in the experiments also provided evidence that the form 2 organisms did not originate from the air or the culture medium. The failure to obtain form 2 organisms from the media 'inoculated' with a sterile loop and aerated during the course of the experiment is against these organisms arising from the air or the medium. The failure to obtain any colonies resembling the form 2 organisms on the plates exposed in the inoculation cabinet during aerations suggest that they did not result from aerial contamination. The failure to obtain form 2 colonies from those slopes which were not aerated (series D, Fig. 1) suggest that they were not medium contaminants. (4) Evidence that contamination arising during aeration of the Löwenstein-Jensen medium cultures was not responsible for the isolation of form 2 organisms is provided furthermore by comparing the frequency with which form 2 colonies were obtained from cultures aerated either by removal of their screw-caps or by allowing air to enter through a cotton-wool plug. Form 2 colonies were obtained from 9 of 30 cultures in groups $\mathbf{A}$ and $\mathbf{B}$, which were aerated by removal of their caps and in 11 of 30 cultures in groups $\mathrm{Al}$ and B1, which were set up in aeration tubes (Fig. 1, Table 1). (5) Although most of the form 2 organisms resemble the genus Bacillus when grown on nutrient agar, all of the isolates from $\boldsymbol{M}$. tuberculosis strains were definitely Gram-negative and it would be remarkable to obtain such a high proportion of Gram-negative Bacillus species. Further experiments, to be described elsewhere, show that the form 2 strains have a complex morphology and life-cycle resembling but not identical with certain species of the families Actinomycetaceae and Streptomycetaceae (Waksman \& Henrici, 1943).

The existence of form 2 organisms in the form 1 cultures used as the inoculum is improbable. The Löwenstein-Jensen medium slopes were inoculated with form 1 cultures which had been purified by single colony selection on two occasions. If a form 2 organism had survived this purification it should have been visible in the smears or should have grown in the cultures on nutrient agar which were made from the inocula in these experiments.

As considered above, it is reasonable to conclude that form 2 organisms were derived from the form 1 cells in certain stages of their life cycle. The exact stage at which the transition between these two forms occurs is uncertain. The serial examinations of Löwenstein-Jensen medium cultures (group C) suggest that in the early phases of their development the form 2 organisms are not able to grow on nutrient agar. In the late phases of their development when spores were present either in an advanced stage in the cells or were lying free, positive isolates were obtained from the spores. These structures were considered as spores since they resemble the spores of different micro-organisms (Bacillaceae, Streptomycetales) in their morphological and staining characteristics and in their capability to survive for longer periods than the vegetative cells. Heat resistance of spores was not investigated, since it is known that spores of Streptomycetales are reproductive bodies rather than resistant bodies and are destroyed by heat at $60-65^{\circ}$ (Waksman, 1950). Spore formation (conidia) in organisms closely related to mycobacteria has been known for a long time. The order Actinomycetales (Waksman \& Henrici, 1943) contains five genera (Mycobacterium, Actinomyces, Nocardia, Streptomyces, Micromonospora) of which two, Streptomyces and Micromonospora have been known for long to form a certain type of spores (conidia), while spores have recently been described in a third genus, Nocardia, by Gordon \& Mihm (1958). The close relationship between the 
genera Mycobacterium, Actinomyces and Nocardia has been commented on by many authors (Jensen, 1931; Skinner, Emmons \& Tsuchiya, 1947; Gordon \& Mihm, 1957; Cummins \& Harris, 1958). Spore formation of Mycobacteria was described and illustrated by Xalabarder (1954) and by Brieger \& Glauert (1956). The latter authors observed round bodies in the filaments of an avian strain of Mycobacterium tuberculosis by electron microscopy; these bodies were identified as spores and had a structure similar to the spores of Bacillus cereus and B. megatherium. The existence in acid-fast mycobacteria cultures of forms that are not acid-fast, is extensive, and has been reviewed by Bassermann (1953) and Koelbel (1957). Rosenthal \& Heagan (1955) described by using bright field, phase and electron microscopy certain cells in the culture of $M$. tuberculosis strain BCG which correspond in bacillary morphology and staining properties to the description of form 2 organisms reported here. They considered that the bacilli which correspond most closely to the early phase 3 described here reverted to normal acid-fast bacilli. In Lack \& Tanner's (1953) paper illustration of organisms in mycobacteria cultures closely resembling the phases 3 and $4 b$ of the present paper are shown in Pl. 3, fig. 10, and Pl. 1, fig. 3. The authors found that these forms were not acid-fast (Dr C. H. Lack, personal communication) except for the middle of the spore-like structures in Pl. 1, fig. 3, which they considered as abnormal nuclei.

Although form 2 organisms differ from form 1 organisms in a variety of important characters such as bacillary morphology, staining characters, colonial morphology, speed of growth and nutritional requirement, they seem to be not two different organisms but two different forms of the same organism. In mycology some species are known to exist in two entirely different forms (dimorphism); this phenomenon is considered by Langeron \& Vanbreuseghem (1952) as a special manifestation of polymorphism. In the pathogenic dimorphic fungi, the morphology of the parasitic form is usually simple but the saprophytic form is complex. For example, Histoplasma capsulatum always grows in the host as a budding yeast, but in vitro it grows either as a yeast, producing smooth, glistening colonies, or reverts to a hyphal type of growth with cottony, mould colonies in which chlamidospores occur regularly. It is reasonable to suggest that the forms 1 and 2 of the mycobacteria described here are examples of such dimorphism. There appears to be no previous report of proved dimorphism in the mycobacteria. Since the form 2 organisms when in phase 4 and when growing on nutrient agar are similar to strains of the genus Bacillus, it would seem probable that form 1 cultures containing them would usually have been discarded as contaminated. The results of attempts to convert form 2 to form 1 mycobacteria and a fuller description of the characteristics of form 2 organisms will be described at a later date.

I wish to express sincere thanks to Dr D. A. Mitchison for his interest in this work and for his severe criticism which nevertheless proved extremely valuable. Our stimulating discussions contributed much to the soundness of the experiments. Finally, I am grateful to him for reading this paper. I am also grateful to $\mathrm{Mr}$ K. Robinson and Miss Janet Lloyd for their conscientious technical assistance. 


\section{REFERENCES}

Bassermann, F. J. (1953). Probleme der Morphologie, Cytochemie und Wuchsform des Tuberculoseerregers, pp. 20, 69. Stuttgart: Georg Thieme Verlag.

Brieger, E. M. \& GlaUert, A . M. (1956). Spore-like structures in the tubercle bacillus. Nature, Lond. 178, 544.

Cohn, M. L., Middlebrook, G. \& Russell, W. F. (1959). Combined drug treatment of tuberculosis. J. clin. Invest. 38, 1349.

Csillag, A. (1960). Periodic acid-Schiff (PAS) staining of 'atypical' mycobacteria and tubercle bacilli. Tubercle, Lond. 41, 63.

Csillag, A. (1961). Morphological and biochemical features of 'atypical' mycobacteria. J. gen. Microbiol. 24, 261.

Cummins, C. S. \& Harris, H. (1958). Studies on the cell-wall composition and taxonomy of Actinomycetales and related groups. J. gen. Microbiol. 18, 173.

Gordon, R. E. \& Minm, J. M. (1957). A comparative study of some strains received as Nocardiae. J. Bact. 73, 15.

Gordon, R. E. \& Minm, J. M. (1958). Sporulation by two strains of Nocardia asteroides. J. Bact. 75, 239.

Jensen, H. L. (1931). Contribution to our knowledge of the Actinomycetales. II. The definition and subdivision of the genus Actinomyces, with a preliminary account of Australian soil actinomycetes. Proc. Linn. Soc. N.S.W. 56, 345.

Jensen, K. A. (1955). Towards a standardisation of laboratory methods. 2nd report of the Subcommittee of laboratory methods of the International Union against Tuberculosis. Bull. int. Un. Tuberc. 25, 89.

Koelbex, H. (1957). Über einige Probleme der Morphologie und Cytologie des Mycobacterium tuberculosis. Jahresbericht Borstel, 1956/57, p. 252. Berlin: Springer Verlag.

LACK, C. H. \& TANNeR, F. (1953). The significance of pleomorphism in Mycobacterium tuberculosis var. hominis. J. gen. Microbiol. 8, 18.

Langeron, M. \& Vanbreuseghem, R. (1952). Précis de Mycologie, 2nd ed., pp. 320, 593. Paris: Masson and Cie.

Mackie \& McCartney's Handbook of Bacteriology (1960). Ed. by R. Cruickshank, pp. 112, 118, 215. Edinburgh and London: Livingstone.

Rosenthal, S. R. \& Heagan, B. (1955). Studies of the life cycle of the tubercle bacillus (BCG). Ann. Inst. Pasteur, 88, 479.

Runyon, E. H. (1959). Anonymous mycobacteria in pulmonary disease. Med. Clin. N. Amer. 43, 273.

Skinner, C. E., Emmons, C. W. \& Tsuchiya, H. M. (1947). Henrici's moulds, yeasts and actinomycetes, p. 354. London: Chapman and Hall.

Tuberculosis Chemotherapy Centre, Madras (1959). A concurrent comparison of home and sanatorium treatment of pulmonary tuberculosis in South India. Bull. Wld Hlth Org. 21, 144.

Waksman, S. A. \& Henrici, A. T. (1943). The nomenclature and classification of the actinomycetes. J. Bact. 46, 337.

Waksman, S. A. (1950). The Actinomycetes, p. 57. Waltham, Mass., U.S.A.: Chronica Botanica Company.

Xalabarder, C. (1954). El origen del Bacilo de Koch, p. 92. Barcelona: Inst. Antituberculoso 'Francisco Moragas'. 

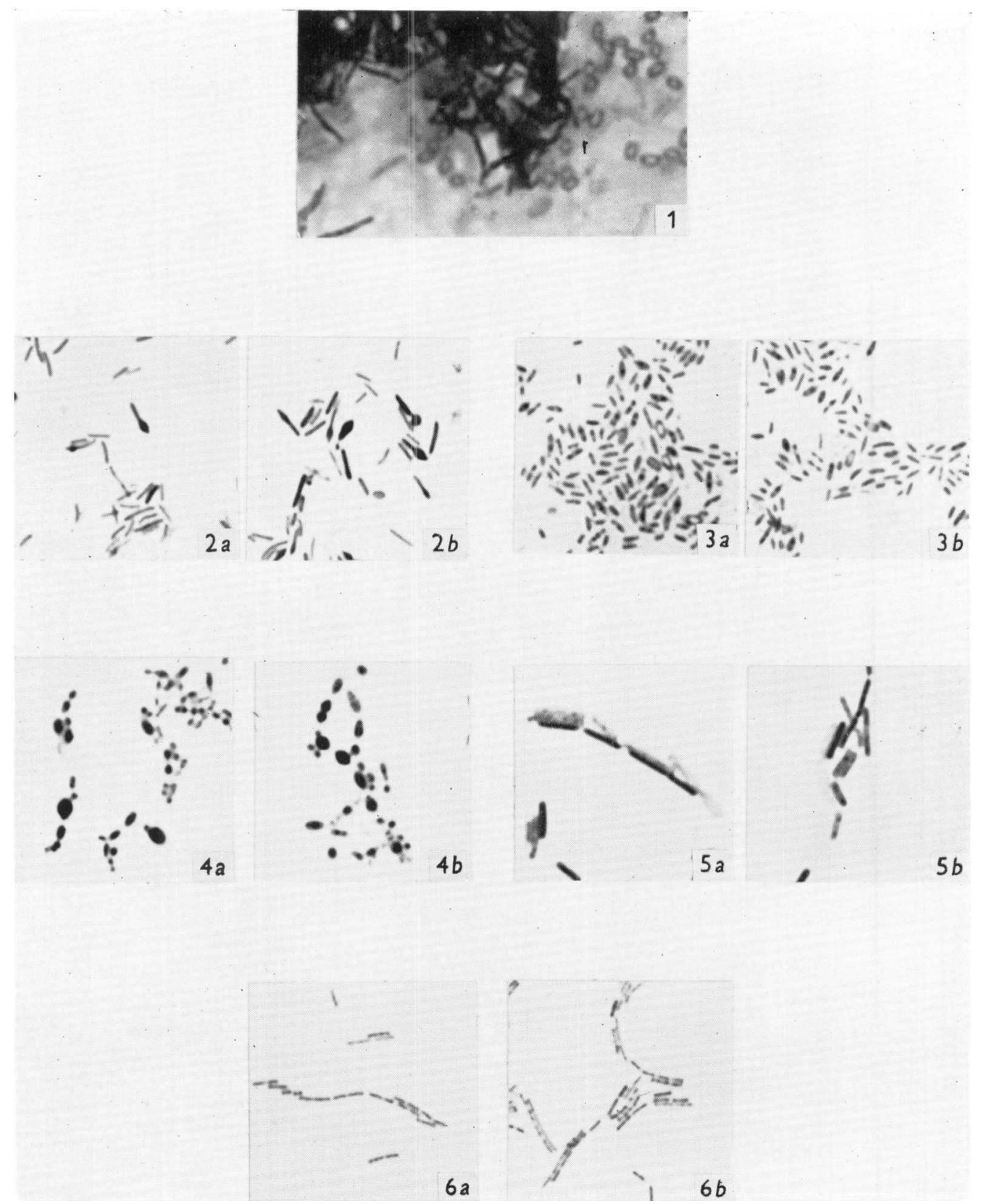

A. Csillag 

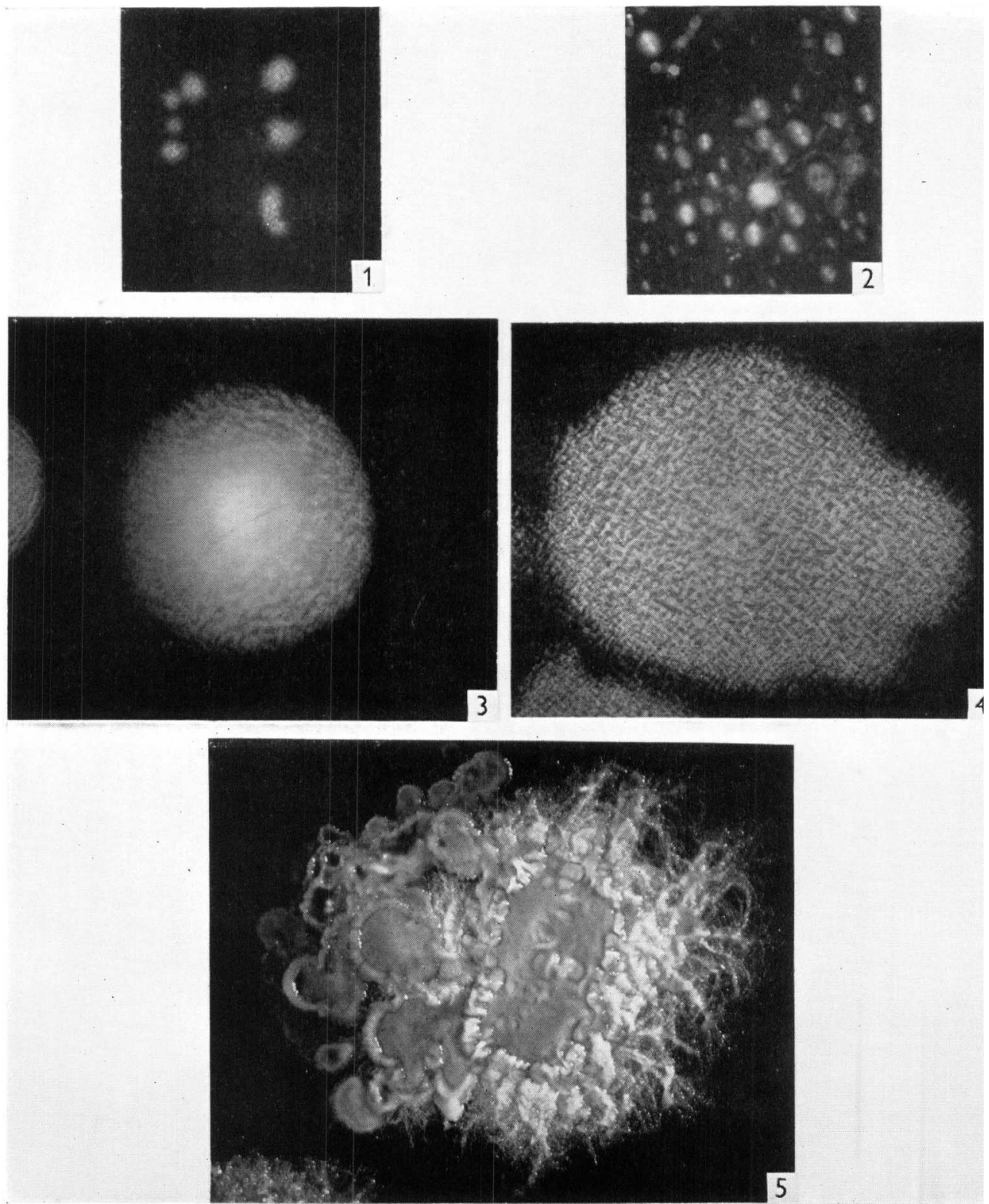

\section{A. CSILLAG}




\section{EXPLANATION OF PLATES}

\section{Plate 1}

Fig. 1. Mycobacterium tuberculosis : 1-6 weeks' incubation with frequent aeration, on LöwensteinJensen medium. Form 1 cells and free spores are shown. Ziehl-Neelsen stain. $\times 2000$.

Figs. 2-6. Form 2 organisms isolated on nutrient agar plates, incubated for 1-5 days. Pairs of photographs of the same strain; the 2 members of the pairs having been obtained in separate groups (Fig. 1). Ziehl-Neelsen stain. $\times 1500$.

Fig. 2a. M. tuberculosis, strain I971, group B.

Fig. 2b. M. tuberculosis, strain I971, group B1.

Fig. 3a. M. tuberculosis, strain I1155, group A.

Fig. 3b. M. tuberculosis, strain I1155, group A1.

Fig. 4a. M. tuberculosis, strain I1133, group A.

Fig. 4b. M. tuberculosis, strain I1133, group A1.

Fig. 5a. Group I, atypical, strain 260, group C.

Fig. 5b. Group I, atypical, strain 260, group $\mathrm{Kg}$.

Fig. 6a. Group I, atypical, strain 1438, group C.

Fig. 6b. Group I, atypical, strain 1438, group C.

\section{Plate 2}

Figs. 1-5. Colonies of form 2 organisms at their first isolation on nutrient agar plates, after 2 days' incubation. $\times 10$.

Fig. 1. M. tuberculosis, strain 1968.

Fig. 2. M. tuberculosis, strain 1971.

Fig. 3. Group I, atypical, strain 260.

Fig. 4. Group I, atypical, strain 1438 .

Fig. 5. Group III, atypical, strain 223. 